\title{
Bilinear approach to quasi-periodic wave solutions of the Kersten-Krasil'shchik coupled KdV-mKdV system
}

\author{
Wenjuan Rui ${ }^{*}$ and Xuemei Qi
}

\author{
"Correspondence: ruiwj@126.com \\ 'College of Science, China \\ University of Mining and \\ Technology, Xuzhou, 221116, \\ P.R. China \\ Full list of author information is \\ available at the end of the article
}

\begin{abstract}
The Hirato bilinear method is extended to construct quasi-periodic wave solutions for the Kersten-Krasil'shchik coupled KdV-mKdV system. One- and two-periodic wave solutions are obtained by means of a multidimensional Riemann theta function. The asymptotic property of the quasi-periodic wave solutions is proved. It is shown that the quasi-periodic wave solutions reduce to the soliton solutions in an asymptotic small amplitude limit.
\end{abstract}

PACS Codes: $02.30 .1 \mathrm{k} ; 02.30 . \mathrm{Gp} ; 05.45 . \mathrm{Yv}$

Keywords: Riemann theta function; soliton solutions; quasi-periodic wave solution

\section{Introduction}

Nonlinear evolution equations (NLEEs) play important roles in many areas of science, and their study goes back a very long time. A variety of effective methods for constructing exact solutions of NLEEs have been developed, including the inverse scattering method, the Darboux transformation, the Hirota direct method, the algebro-geometrical approach [1-5], etc. Among the aforementioned methods, the Hirota method has proved particularly powerful. Once the corresponding bilinear form is derived, multi-soliton solutions for a nonlinear equation are usually obtained in a systematic way. Recently, many generalizations of the Hirota method were developed. For example, a multiple exp-function method is proposed and provides an efficient way to an exact multiple wave solution procedure that generalizes Hirota's perturbation technique $[6,7]$. The resulting multiple wave solutions contain resonant solitons and a linear combination of solutions of exponential waves, the latter of which presents an idea to construct linear subspaces of solutions for nonlinear equations. The Hirota bilinear method has been generalized to a broad class of nonlinear equations [8] and the links with Bell polynomials were established [9]. It is interesting to note that the linear superposition principle can also apply to Hirota bilinear equations and present their resonant soliton solutions [10], which is a special case of using the Riemann theta functions. Based on the Hitota bilinear method, Nakamura proposed an approach to quasi-periodic wave solutions of nonlinear equations [11, 12]. This method uses the multidimensional Riemann theta function directly and does not involve Lax pair representations and the complicated calculus on Riemann surfaces for the equations con-

(c) 2016 Rui and Qi. This article is distributed under the terms of the Creative Commons Attribution 4.0 International License (http://creativecommons.org/licenses/by/4.0/), which permits unrestricted use, distribution, and reproduction in any medium, provided you give appropriate credit to the original author(s) and the source, provide a link to the Creative Commons license, and indicate if changes were made. 
sidered. Recently, Fan, Hon, Ma, and Zhang have extended such method to investigate the breaking soliton equation, the discrete Toda lattice, the asymmetrical Nizhnik-NovikovVeselov equation and the variable-coefficient $\mathrm{mKdV}$ equation, respectively [13-18].

In this paper, we would like to consider the following coupled KdV-mKdV system:

$$
\begin{aligned}
& u_{t}+u_{x x x}-6 u u_{x}+3 v v_{x x x}+3 v_{x} v_{x x}-3 u_{x} v^{2}-6 u v v_{x}=0, \\
& v_{t}+v_{x x x}-3 v^{2} v_{x}-3 u v_{x}-3 u_{x} v=0
\end{aligned}
$$

which was proposed as the classical part of one of superextensions of the KdV equation by Kersten and Krasil'shchik [19]. Taking $v=0$, we have the KdV equation

$$
u_{t}+u_{x x x}-6 u u_{x}=0
$$

while for $u=0$ we get the $\mathrm{mKdV}$ equation

$$
v_{t}+v_{x x x}-3 v^{2} v_{x}=0
$$

Therefore, system (1.1) can also be regarded as a type of coupling between the KdV and the mKdV equations. For system (1.1), Kersten and Krasil'shchik proved its complete integrability by establishing the existence of an infinite series of symmetries and conservation laws [19]; Kalkanli et al. presented its singular analysis and Lax pair by means of the Painlevé test and prolongation technique [20]; Hon and Fan devised an algebraic method to construct its solitary wave solutions and doubly periodic wave solutions [21]; Qin et al. applied the Bell polynomials to derive its bilinear form and $N$-soliton solutions [22]. However, to the best of our knowledge, the quasi-periodic wave solutions for the system (1.1) have not been investigated. In the following, the Riemann theta function and the Hirato bilinear method will be applied to construct one- and two-periodic wave solutions for the system (1.1). In addition, a detailed asymptotic analysis procedure to the quasi-periodic wave solutions is presented and the relation between the quasi-periodic wave solutions and soliton solutions is established.

This paper is organized as follows. In Section 2, we briefly introduce some main points on the Riemann theta function. In Section 3 and Section 4, we construct one- and twoperiodic wave solutions for the coupled KdV-mKdV system based on the Riemann theta function and bilinear method. Furthermore, the relation between the quasi-periodic wave solutions and soliton solutions is investigated. Finally, some concluding remarks are given in Section 5.

\section{The bilinear form and the Riemann theta function}

To begin with, we introduce a useful bilinear form of system (1.1). Under the dependent variable transformation defined by [22]

$$
u=-\partial_{x}^{2} \ln \left(f f^{*}\right), \quad v=i \partial_{x} \ln \frac{f^{*}}{f}
$$


where $f^{*}$ is the conjugation of $f$, system (1.1) is transformed into the following bilinear form:

$$
\begin{aligned}
& \left(D_{t}+D_{x}^{3}\right) f \cdot f^{*}=0, \\
& \left(D_{x} D_{t}+D_{x}^{4}\right) f \cdot f^{*}=0 .
\end{aligned}
$$

Following the Hirota bilinear method, the one-soliton solution for system (1.1) is of the form

$$
u_{1}=-\partial_{x}^{2} \ln \left(1+e^{2 \eta}\right), \quad v_{1}=i \partial_{x} \ln \frac{1-i e^{\eta}}{1+i e^{\eta}}
$$

where $\eta=\alpha x-\alpha^{3} t+\sigma$ and $\alpha, \sigma$ are free constants. The two-soliton solution for system (1.1) is denoted by

$$
\begin{aligned}
& u_{2}=-\partial_{x}^{2} \ln \left[\left(1-e^{\eta_{1}+\eta_{2}+A_{12}}\right)^{2}+\left(e^{\eta_{1}}+e^{\eta_{2}}\right)^{2}\right], \\
& v_{2}=i \partial_{x} \ln \frac{1-i e^{\eta_{1}}-i e^{\eta_{2}}-e^{\eta_{1}+\eta_{2}+A_{12}}}{1+i e^{\eta_{1}}+i e^{\eta_{2}}-e^{\eta_{1}+\eta_{2}+A_{12}}},
\end{aligned}
$$

with

$$
\begin{aligned}
& \eta_{j}=\alpha_{j} x-\alpha_{j}^{3} t+\sigma_{j}, \quad j=1,2, \\
& e^{A_{12}}=\left(\frac{\alpha_{1}-\alpha_{2}}{\alpha_{1}+\alpha_{2}}\right)^{2},
\end{aligned}
$$

and here $\alpha_{j}, \sigma_{j}$ are constants.

To construct quasi-periodic wave solutions of system (1.1), it is necessary for us to consider a generalized form of the bilinear equation (2.2). Assume the solution of (1.1) takes the form

$$
u=u_{0}-\partial_{x}^{2} \ln \left(f^{*}\right), \quad v=i \partial_{x} \ln \frac{f^{*}}{f}
$$

where $u_{0}$ is a constant solution of (1.1). Submitting (2.5) into (1.1), we get

$$
\begin{aligned}
& H_{1}\left(D_{x}, D_{t}\right) f \cdot f^{*}=\left(D_{t}+D_{x}^{3}-3 u_{0} D_{x}\right) f \cdot f^{*}=0, \\
& H_{2}\left(D_{x}, D_{t}\right) f \cdot f^{*}=\left(D_{x} D_{t}+D_{x}^{4}-6 u_{0} D_{x}^{2}+c\right) f \cdot f^{*}=0,
\end{aligned}
$$

where $c=c(t)$ is an integration constant. The constant $c$ is vital in the construction of quasi-periodic solutions because elliptic functions do not usually satisfy equations with zero integration constants.

In the following, we will construct quasi-periodic solutions for the bilinear equation (2.6) based on the multidimensional Riemann theta function [23]

$$
\vartheta(\xi, \epsilon, s \mid \tau)=\sum_{n \in Z^{N}} e^{-\pi\langle\tau(n+s), n+s\rangle+2 \pi i\langle\xi+\epsilon, n+s\rangle},
$$

where $\langle\cdot\rangle$ is the standard inner product of $R^{n}, n=\left(n_{1}, \ldots, n_{N}\right)^{T} \in Z^{N}$, we have the complex phase variable $\xi=\left(\xi_{1}, \ldots, \xi_{N}\right)^{T} \in C^{N}$, the complex parameter vector $s=\left(s_{1}, \ldots, s_{N}\right)^{T}, \epsilon=$ 
$\left(\epsilon_{1}, \ldots, \epsilon_{N}\right)^{T}$, and the matrix $\tau=\left(\tau_{i j}\right)$ is a positive definite and real-valued symmetric $N \times N$ matrix.

In the definition of the Riemann theta function, the positive definiteness of $\tau$ guarantees (2.7) converges absolutely for all values of $\xi$. For simplicity, hereafter we use $\vartheta(\xi+\epsilon, \tau)=$ $\vartheta(\xi, \epsilon, 0 \mid \tau)$. Now let us see the periodicity of the Riemann theta function.

Definition 1 A function $g(x, t)$ on $C^{N} \times C$ is said to be quasi-periodic in $t$ with fundamental periods $T_{1}, \ldots, T_{k} \in C$ if $T_{1}, \ldots, T_{k}$ are linearly dependent over $Z$ and there exists a function $G(t, t) \in C^{N} \times C^{k}$ such that for $\forall\left(y_{1}, \ldots, y_{k}\right) \in C^{k}$

$$
\begin{aligned}
& G\left(x, y_{1}, \ldots, y_{j}+T_{j}, \ldots, y_{k}\right)=G\left(x, y_{1}, \ldots, y_{j}, \ldots, y_{k}\right), \\
& G(x, t, \ldots, t, \ldots, t)=g(x, t) .
\end{aligned}
$$

In particular, $g(x, t)$ becomes periodic with $T$ if and only if $T_{j}=m_{j} T$.

Proposition 1 [24] Let $e_{j}$ be the jth column of $N \times N$ identity matrix $I_{N}, \tau_{j}$ the jth column of $\tau$, and $\tau_{j j}$ the $(j, j)$ entry of $\tau$. Then the theta function $\vartheta(\xi, \tau)$ has the periodic properties

$$
\vartheta\left(\xi+e_{j}+i \tau_{j}, \tau\right)=\exp \left(-2 \pi i \xi_{j}+\pi \tau_{j j}\right) \vartheta(\xi, \tau)
$$

The vectors $\left\{e_{j}, j=1, \ldots, N\right\}$ and $\left\{i \tau_{j}, j=1, \ldots, N\right\}$ can be regarded as periods of the theta function $\vartheta(\xi, \tau)$ with multipliers 1 and $\exp \left(-2 \pi i \xi_{j}+\pi \tau_{j j}\right)$, respectively.

In general, let $\vartheta\left(\xi, \epsilon^{\prime}, 0 \mid \tau\right)$ and $\vartheta(\xi, \epsilon, 0 \mid \tau)$ be two Riemann theta functions, where $\epsilon=$ $\left(\epsilon_{1}, \ldots, \epsilon_{N}\right)^{T}, \epsilon^{\prime}=\left(\epsilon_{1}^{\prime}, \ldots, \epsilon_{N}^{\prime}\right)^{T}$, and $\xi=\left(\xi_{1}, \ldots, \xi_{N}\right)^{T}, \xi_{j}=k_{j} x+l_{j} y+m_{j} t+\gamma_{j}, j=1,2, \ldots, N$. For a polynomial operator $H\left(D_{x}, D_{y}, D_{t}\right)$ with respect to $D_{x}, D_{y}$, and $D_{t}$, the following formula holds:

$$
\begin{aligned}
& H\left(D_{x}, D_{y}, D_{t}\right) \vartheta\left(\xi, \epsilon^{\prime}, 0 \mid \tau\right) \cdot \vartheta(\xi, \epsilon, 0 \mid \tau) \\
& \quad=\sum_{\mu} C\left(\epsilon^{\prime}, \epsilon, \mu\right) \vartheta\left(2 \xi, \epsilon^{\prime}+\epsilon, \mu / 2 \mid 2 \tau\right),
\end{aligned}
$$

where

$$
\begin{aligned}
C\left(\epsilon^{\prime}, \epsilon, \mu\right)= & \sum_{n \in Z^{N}} H(4 \pi i\langle n-\mu / 2, k\rangle, 4 \pi i\langle n-\mu / 2, l\rangle, 4 \pi i\langle n-\mu / 2, m\rangle) \\
& \times \exp \left\{-2 \pi\langle\tau(n-\mu / 2), n-\mu / 2\rangle+2 \pi i\left\langle n-\mu / 2, \epsilon^{\prime}-\epsilon\right\rangle\right\} .
\end{aligned}
$$

From equations (2.8) and (2.9), we see that if

$$
C\left(\epsilon^{\prime}, \epsilon, \mu\right)=0
$$

are satisfied for all possible combinations $\mu_{1}=0,1 ; \ldots, \mu_{N}=0,1$, then $\vartheta\left(\xi, \epsilon^{\prime}, 0 \mid \tau\right)$ and $\vartheta(\xi, \epsilon, 0 \mid \tau)$ are quasi-periodic solutions of the bilinear equation

$$
H\left(D_{x}, D_{y}, D_{t}\right) \vartheta\left(\xi, \epsilon^{\prime}, 0 \mid \tau\right) \cdot \vartheta(\xi, \epsilon, 0 \mid \tau)=0 .
$$


The coupled bilinear equation (2.6) is more difficult to deal with than a single bilinear equation due to the appearance of two equations. The following proposition plays an important role in constructing its quasi-periodic wave solutions.

Proposition 2 [16] Let $C(\mu)$ be given in (2.9), and make a choice such that $\epsilon_{j}^{\prime}-\epsilon_{j}= \pm \frac{1}{2}$, $j=1,2, \ldots, N$. Then:

(1) If $H\left(D_{x}, D_{y}, D_{t}\right)$ is an even function of the form

$$
H\left(-D_{x},-D_{y},-D_{t}\right)=H\left(D_{x}, D_{y}, D_{t}\right)
$$

then $C(\mu)$ vanishes automatically for the case when $\sum_{j=1}^{N} \mu_{j}$ is an odd number, namely

$$
\left.C(\mu)\right|_{\mu}=0, \quad \text { for } \sum_{j=1}^{N} \mu_{j}=1 \quad \bmod 2
$$

(2) If $H\left(D_{x}, D_{y}, D_{t}\right)$ is an odd function of the form

$$
H\left(-D_{x},-D_{y},-D_{t}\right)=-H\left(D_{x}, D_{y}, D_{t}\right),
$$

then $C(\mu)$ vanishes automatically for the case when $\sum_{j=1}^{N} \mu_{j}$ is an even number, namely

$$
\left.C(\mu)\right|_{\mu}=0, \quad \text { for } \sum_{j=1}^{N} \mu_{j}=0 \quad \bmod 2 \text {. }
$$

\section{One-periodic wave solutions and asymptotic properties}

In this section, we construct the one-periodic wave solutions for the system (1.1) with $N=1$ in Riemann theta function (2.7). Setting $f=\vartheta\left(\xi, \frac{1}{4}, 0 \mid \tau\right)$ and $f^{*}=\vartheta\left(\xi, \frac{3}{4}, 0 \mid \tau\right), f$ and $f^{*}$ can be written as the following Fourier series in $n$ :

$$
\begin{aligned}
& f=\vartheta\left(\xi, \frac{1}{4}, 0 \mid \tau\right)=\sum_{n \in Z} i^{n} e^{2 \pi i n \xi-\pi n^{2} \tau}, \\
& f^{*}=\vartheta\left(\xi, \frac{3}{4}, 0 \mid \tau\right)=\sum_{n \in Z}(-i)^{n} e^{2 \pi i n \xi-\pi n^{2} \tau},
\end{aligned}
$$

where the phase variable $\xi=k x+l t+\gamma$, and the parameter $\tau>0$.

\subsection{Construction of one-periodic wave solution}

In order to get the one-periodic wave solutions of the bilinear equation (2.6), we substitute (3.1) into (2.6), thus for $i=1,2$

$$
\begin{aligned}
& H_{i}\left(D_{x}, D_{t}\right) f \cdot f^{*} \\
& \quad=\sum_{\mu}\left[\sum_{n \in Z} H_{i}\left(4 \pi i\left\langle n-\frac{\mu}{2}, k\right\rangle, 4 \pi i\left\langle n-\frac{\mu}{2}, l\right\rangle\right)\right.
\end{aligned}
$$




$$
\begin{aligned}
& \left.\quad \times \exp \left\{-2 \pi\left\langle\tau\left(n-\frac{\mu}{2}\right), n-\frac{\mu}{2}\right\rangle+2 \pi i\left\langle n-\frac{\mu}{2}, \epsilon^{\prime}-\epsilon\right\rangle\right\}\right] \\
& \times \vartheta\left(2 \xi, 1, \frac{\mu}{2} \mid 2 \tau\right) \\
& =\sum_{\mu} C_{i}(\mu) \vartheta\left(2 \xi, 1, \frac{\mu}{2} \mid 2 \tau\right), \quad \mu=0,1,
\end{aligned}
$$

where

$$
\begin{aligned}
C_{1}(\mu)= & \sum_{n \in Z}\left[4 \pi i\left(n-\frac{\mu}{2}\right) l-64 \pi^{3} i\left(n-\frac{\mu}{2}\right)^{3} k^{3}-12 \pi i\left(n-\frac{\mu}{2}\right) u_{0} k\right] \\
& \times \exp \left\{-2 \pi \tau\left(n-\frac{\mu}{2}\right)^{2}+\pi i\left(n-\frac{\mu}{2}\right)\right\}, \\
C_{2}(\mu)= & \sum_{n \in Z}\left[-16 \pi^{2}\left(n-\frac{\mu}{2}\right)^{2} k l+256 \pi^{4}\left(n-\frac{\mu}{2}\right)^{4} k^{4}+96 \pi^{2}\left(n-\frac{\mu}{2}\right)^{2} u_{0} k^{2}+c\right] \\
& \times \exp \left\{-2 \pi \tau\left(n-\frac{\mu}{2}\right)^{2}+\pi i\left(n-\frac{\mu}{2}\right)\right\} .
\end{aligned}
$$

According to Proposition 2, due to the fact $H_{1}\left(D_{x}, D_{t}\right)$ is an odd function, we have $C_{1}(\mu=$ $0)=0$. Meanwhile, $H_{2}\left(D_{x}, D_{t}\right)$ is an even function and $C_{2}(\mu=1)=0$. Therefore, if the following equations are satisfied:

$$
\begin{aligned}
C_{1}(\mu=1)= & \sum_{n \in Z}\left[4 \pi i\left(n-\frac{1}{2}\right) l-64 \pi^{3} i\left(n-\frac{1}{2}\right)^{3} k^{3}-12 \pi i\left(n-\frac{1}{2}\right) u_{0} k\right] \\
& \times \exp \left\{-2 \pi \tau\left(n-\frac{1}{2}\right)^{2}+\pi i\left(n-\frac{1}{2}\right)\right\}=0 \\
C_{2}(\mu=0)= & \sum_{n \in Z}\left[-16 \pi^{2} n^{2} k l+256 \pi^{4} n^{4} k^{4}+96 \pi^{2} n^{2} u_{0} k^{2}+c\right] \\
& \times \exp \left\{-2 \pi \tau n^{2}+\pi i n\right\}=0
\end{aligned}
$$

the Riemann theta functions (3.1) are exact solutions of equation (2.6). Taking $\rho, \vartheta_{1}, \vartheta_{2}$ of the form

$$
\begin{aligned}
& \rho=e^{-\frac{\pi \tau}{2}} \\
& \begin{aligned}
\vartheta_{1}(\xi, \rho) & =\vartheta\left(2 \xi, \frac{1}{2},-\frac{1}{2} \mid 2 \tau\right) \\
& =\sum_{n \in Z} \rho^{(2 n-1)^{2}} \exp \left[4 \pi i\left(n-\frac{1}{2}\right)\left(\xi+\frac{1}{4}\right)\right], \\
\vartheta_{2}(\xi, \rho) & =\vartheta\left(2 \xi, \frac{1}{2}, 0 \mid 2 \tau\right) \\
& =\sum_{n \in Z} \rho^{4 n^{2}} \exp \left[4 \pi i n\left(\xi+\frac{1}{4}\right)\right]
\end{aligned}
\end{aligned}
$$




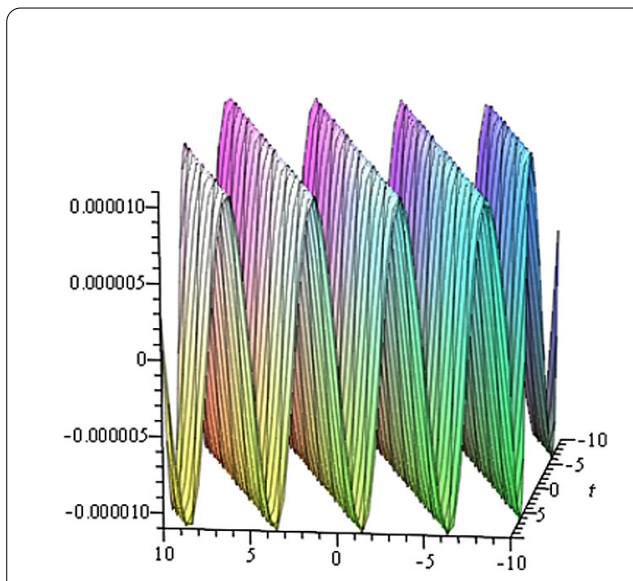

(a)

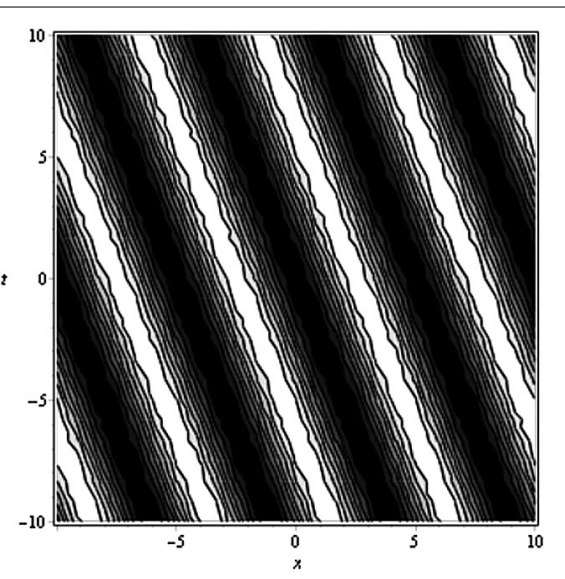

(b)

Figure 1 One-periodic wave of $u$ with parameters: $k=0.1, \gamma=0, \tau=2, u_{0}=0$. (a) Perspective view of the wave. (b) Overhead view of the wave, with contour plot shown.

we transform equation (3.2) into a linear system about $l$ and $c$

$$
\begin{aligned}
& \vartheta_{1}^{\prime} l+\vartheta_{1}^{\prime \prime \prime} k^{3}-3 u_{0} \vartheta_{1}^{\prime} k=0, \\
& \vartheta_{2}^{\prime \prime} k l+\vartheta_{2}^{(4)} k^{4}-6 u_{0} \vartheta_{2}^{\prime \prime} k^{2}+c \vartheta_{2}=0,
\end{aligned}
$$

where

$$
\vartheta_{j}^{(p)}=\vartheta_{j}^{(p)}(0, \rho)=\left.\frac{d \vartheta_{j}^{(p)}}{d \xi}\right|_{\xi=0}, \quad j=1,2 ; p=0,1,2,3,4
$$

Solving equation (3.4), we get

$$
l=-\frac{\vartheta_{1}^{\prime \prime \prime}}{\vartheta_{1}^{\prime}} k^{3}+3 u_{0} k, \quad c=\frac{\vartheta_{1}^{\prime \prime \prime} \vartheta_{2}^{\prime \prime}-\vartheta_{2}^{(4)} \vartheta_{1}^{\prime}}{\vartheta_{1}^{\prime} \vartheta_{2}} k^{4}+\frac{3 u_{0} \vartheta_{2}^{\prime \prime}}{\vartheta_{2}} k^{2}
$$

In this way, we get a one-periodic wave solution of system (1.1),

$$
u=u_{0}-\partial_{x}^{2} \ln \left(\vartheta\left(\xi, \frac{1}{4}, 0 \mid \tau\right) \vartheta\left(\xi, \frac{3}{4}, 0 \mid \tau\right)\right), \quad v=i \partial_{x} \ln \frac{\vartheta\left(\xi, \frac{3}{4}, 0 \mid \tau\right)}{\vartheta\left(\xi, \frac{1}{4}, 0 \mid \tau\right)}
$$

where $\xi=k x+l t+\gamma, l$ is given by (3.5) and the other parameters, $k, \tau, \gamma$, and $u_{0}$, are free. A one-periodic wave solution is determined by the two parameters $k$ and $\tau$. Figures 1 and 2 show one-periodic waves of $u$ and $v$ for one choice of the parameters, respectively.

From Figures 1 and 2, one can see that the one-periodic wave solution of system (1.1) is one dimensional and has two fundamental periods, 1 and $i \tau$, in $\xi$. It can be viewed as a parallel superposition of overlapping one-solitary waves, placed one period apart.

\subsection{Asymptotic property of one-periodic waves}

In the following, we will investigate the asymptotic properties of the one-periodic wave solution (3.6). The relation between the one-soliton solution (2.3) and the one-periodic wave solution (3.6) is given by the following theorem. 


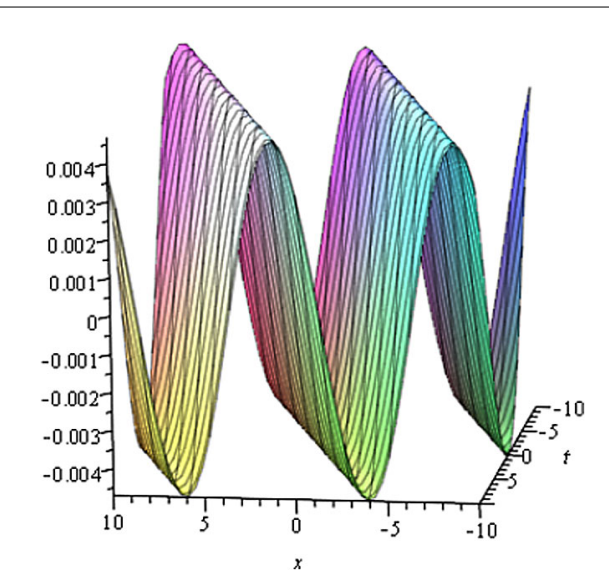

(a)

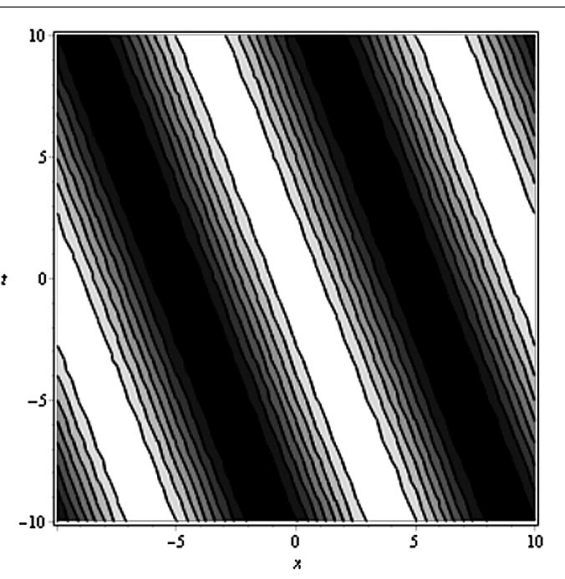

(b)

Figure 2 One-periodic wave of $v$ with parameters: $k=0.1, \gamma=0, \tau=2$. (a) Perspective view of the wave. (b) Overhead view of the wave, with contour plot shown.

Theorem 1 Suppose that $(l, c)^{T}$ is a solution of equation (3.4), let

$$
u_{0}=0, \quad \gamma=\frac{\sigma}{2 \pi i}+\frac{\tau}{2 i}, \quad k=\frac{\alpha}{2 \pi i},
$$

in which $\alpha$ and $\sigma$ are the same as those in (2.3). Then the one-periodic solution (3.6) tends to the one-soliton solution (2.3), namely

$$
u \rightarrow u_{1}, \quad v \rightarrow v_{1}, \quad \text { as } \rho \rightarrow 0
$$

Proof We expand the coefficients of system (3.4) into power series of $\rho$

$$
\begin{aligned}
& \vartheta_{1}^{\prime}=-4 \pi \rho+12 \pi \rho^{9}+\cdots, \quad \vartheta_{1}^{\prime \prime \prime}=16 \pi^{3} \rho+432 \pi^{3} \rho^{9}+\cdots, \\
& \vartheta_{2}=1-2 \rho^{4}+\cdots, \quad \vartheta_{2}^{\prime \prime}=32 \pi^{2} \rho^{4}-128 \pi^{2} \rho^{16}+\cdots, \\
& \vartheta_{2}^{(4)}=-512 \pi^{4} \rho^{4}+8,192 \pi^{4} \rho^{16}+\cdots .
\end{aligned}
$$

Assume that the solution of system (3.4) takes the form

$$
\begin{aligned}
& l=l_{0}+l_{1} \rho+l_{2} \rho^{2}+\cdots, \\
& c=c_{0}+c_{1} \rho+c_{2} \rho^{2}+\cdots,
\end{aligned}
$$

substituting equations (3.8) and (3.9) into equation (3.4) and letting $\rho \rightarrow 0$, we get

$$
l_{0}=4 \pi^{2} k^{3}, \quad c_{0}=0 .
$$

From (3.7) and (3.10), we have

$$
\begin{aligned}
& c \rightarrow 0, \quad 2 \pi i l \rightarrow 8 \pi^{3} k^{3} i=-\alpha^{3}, \quad \text { as } \rho \rightarrow 0, \\
& \xi^{\prime}=2 \pi i \xi-\pi \tau=\alpha x+2 \pi i l t+\sigma \\
& \rightarrow \alpha x-\alpha^{3} t+\sigma=\eta, \quad \text { as } \rho \rightarrow 0 .
\end{aligned}
$$


In order to verify that the one-periodic wave (3.6) tends to the one-soliton solution (2.3) when $\rho \rightarrow 0$, we expand the function $f$ in the form

$$
f=1+\rho^{2}\left(i e^{2 \pi i \xi}-i e^{-2 \pi i \xi}\right)-\rho^{8}\left(e^{4 \pi i \xi}+e^{-4 \pi i \xi}\right)+\cdots
$$

It follows from (3.11) that

$$
\begin{aligned}
f & =1+i e^{\xi^{\prime}}-\rho^{4}\left(e^{2 \xi^{\prime}}+i e^{-\xi^{\prime}}\right)+\rho^{12}\left(e^{3 \xi^{\prime}}+e^{-2 \xi^{\prime}}\right)+\cdots \\
& \rightarrow 1+i e^{\eta}, \quad \text { as } \rho \rightarrow 0
\end{aligned}
$$

Therefore,

$$
f^{*} \rightarrow 1-i e^{\eta}, \quad \text { as } \rho \rightarrow 0 .
$$

we conclude that the one-periodic solution (3.6) tends to one-soliton solution (2.3) when $\rho \rightarrow 0$.

\section{Two-periodic wave solutions and asymptotic properties}

Let us consider two-periodic wave solutions for the coupled KdV-mKdV system (1.1). In the case of $N=2$, we take $f$ and $f^{*}$ as

$$
\begin{aligned}
f & =\vartheta(\xi, \epsilon, 0 \mid \tau) \\
& =\sum_{n \in Z^{2}} e^{-\pi\langle\tau n, n\rangle+2 \pi i\langle\xi+\epsilon, n\rangle} \\
& =\sum_{n \in Z^{2}}(i)^{n_{1}+n_{2}} e^{-\pi\langle\tau n, n\rangle+2 \pi i\langle\xi, n\rangle}, \\
f^{*} & =\vartheta\left(\xi, \epsilon^{\prime}, 0 \mid \tau\right) \\
& =\sum_{n \in Z^{2}} e^{-\pi\langle\tau n, n\rangle+2 \pi i\left\langle\xi+\epsilon^{\prime}, n\right\rangle}, \\
& =\sum_{n \in Z^{2}}(-i)^{n_{1}+n_{2}} e^{-\pi\langle\tau n, n\rangle+2 \pi i\langle\xi, n\rangle},
\end{aligned}
$$

where $n=\left(n_{1}, n_{2}\right)^{T} \in Z^{2}, \xi=\left(\xi_{1}, \xi_{2}\right)^{T} \in C^{2}, \xi_{j}=k_{j} x+l_{j} t+\gamma_{j}, j=1,2, k=\left(k_{1}, k_{2}\right)^{T}, l=\left(l_{1}, l_{2}\right)^{T}$, $\epsilon=\left(\frac{1}{4}, \frac{1}{4}\right)^{T}, \epsilon^{\prime}=\left(\frac{3}{4}, \frac{3}{4}\right)^{T} . \tau$ is a positive definite and real-valued symmetric $2 \times 2$ matrix, which can be written as

$$
\tau=\left(\tau_{p q}\right)_{2 \times 2}, \quad \tau_{11}>0, \tau_{22}>0, \tau_{11} \tau_{22}-\tau_{12}^{2}>0 .
$$

\subsection{Construction of two-periodic waves}

By means of Proposition 2, noting that the fact that $H_{1}\left(D_{x}, D_{t}\right)$ is an odd function, its corresponding constraint equations in (2.10) vanish automatically for $\mu=(0,0),(1,1)$. Similarly, the constraint equations of $H_{2}\left(D_{x}, D_{t}\right)$ vanish for $\mu=(1,0),(0,1)$. Therefore, the theta 
functions (4.1) are solutions of the bilinear equation (2.6) if the following equations:

$$
\begin{aligned}
& \sum_{n \in Z^{2}}\left[4 \pi i\left\langle n-\frac{\mu}{2}, l\right\rangle-64 \pi^{3} i\left\langle n-\frac{\mu}{2}, k\right\rangle^{3}-12 u_{0} \pi i\left\langle n-\frac{\mu}{2}, k\right\rangle\right] \\
& \quad \times\left.\exp \left\{-2 \pi\left\langle\tau\left(n-\frac{\mu}{2}\right), n-\frac{\mu}{2}\right\rangle+2 \pi i\left\langle n-\frac{\mu}{2}, \epsilon^{\prime}-\epsilon\right\rangle\right\}\right|_{\mu=(1,0),(0,1)}=0, \\
& \sum_{n \in Z^{2}}\left[-16 \pi^{2}\left\langle n-\frac{\mu}{2}, k\right\rangle\left\langle n-\frac{\mu}{2}, l\right\rangle+256 \pi^{4}\left\langle n-\frac{\mu}{2}, k\right\rangle\right. \\
& \left.+96 u_{0} \pi^{2}\left\langle n-\frac{\mu}{2}, k\right\rangle^{2}+c\right] \\
& \quad \times\left.\exp \left\{-2 \pi\left\langle\tau\left(n-\frac{\mu}{2}\right), n-\frac{\mu}{2}\right\rangle+2 \pi i\left\langle n-\frac{\mu}{2}, \epsilon^{\prime}-\epsilon\right\rangle\right\}\right|_{\mu=(1,1),(0,0)}=0,
\end{aligned}
$$

are satisfied. Taking

$$
\begin{aligned}
& \rho_{p q}=e^{-\frac{\pi \tau p q}{2}}, \quad p, q=1,2, \\
& \begin{aligned}
& \rho=\left(\rho_{11}, \rho_{12}, \rho_{22}\right) \\
& \vartheta_{r}(\xi, \rho)=\vartheta\left(2 \xi, \epsilon^{\prime}-\epsilon,-\frac{s_{r}}{2} \mid 2 \tau\right) \\
&=\sum_{n \in Z^{2}} \exp \left[-2 \pi\left\langle\tau\left(n-\frac{s_{r}}{2}\right), n-\frac{s_{r}}{2}\right\rangle+2 \pi i\left\langle 2 \xi+\epsilon^{\prime}-\epsilon, n-\frac{s_{r}}{2}\right\rangle\right] . \\
&=\sum_{n \in Z^{2}} \exp \left[4 \pi i\left\langle\xi+\frac{\epsilon^{\prime}-\epsilon}{2}, n-\frac{s_{r}}{2}\right\rangle\right] \prod_{p, q=1}^{2} \rho_{p q}^{\left(2 n_{p}-s_{r, p}\right)\left(2 n_{q}-s_{r, q}\right)}, \\
& s_{r}=\left(s_{r 1}, s_{r 2}\right), \quad r=1,2,3,4, s_{2}=(0,1), \quad s_{3}=(1,1), \quad s_{4}=(0,0),
\end{aligned}
\end{aligned}
$$

equation (4.2) can be transformed into a linear system about $l_{1}, l_{2}, c$, and $u_{0}$,

$$
\begin{aligned}
& (l \cdot \nabla) \vartheta_{r}+(k \cdot \nabla)^{3} \vartheta_{r}-\left.3 u_{0}(k \cdot \nabla) \vartheta_{r}\right|_{\left(\xi_{1}, \xi_{2}\right)=(0,0)}=0, \quad r=1,2, \\
& (k \cdot \nabla)(l \cdot \nabla) \vartheta_{r}+(k \cdot \nabla)^{4} \vartheta_{r}-6 u_{0}(k \cdot \nabla)^{2} \vartheta_{r}+\left.c \vartheta_{r}\right|_{\left(\xi_{1}, \xi_{2}\right)=(0,0)}=0, \quad r=3,4,
\end{aligned}
$$

where $\nabla=\left(\partial \xi_{1}, \partial \xi_{2}\right), k \cdot \nabla=k_{1} \partial \xi_{1}+k_{2} \partial \xi_{2}$. Solving equation (4.4), we can get a twoperiodic wave solution of the system (1.1)

$$
u=u_{0}-\partial_{x}^{2} \ln \left(f^{*}\right), \quad v=i \partial_{x} \ln \frac{f^{*}}{f}
$$

where $f, f^{*}$, and $l_{1}, l_{2}, c, u_{0}$ are given by equations (4.1) and (4.4), and the other parameters, $k_{1}, k_{2}, \tau_{11}, \tau_{12}, \tau_{22}$, are free. A two-periodic wave solution is specified by the four parameters $k_{1}, k_{2}, \tau_{11}$, and $\tau_{22}$. 


\subsection{Asymptotic property of two-periodic waves}

Similar to Theorem 1, the relation between the two-soliton solution (2.4) and the twoperiodic solution (4.5) can be given as follows.

Theorem 2 Suppose that $\left(l_{1}, l_{2}, u_{0}, c\right)^{T}$ is a solution of system (4.4), let

$$
\begin{aligned}
& \gamma_{j}=\frac{\sigma_{j}}{2 \pi i}+\frac{\tau_{j j}}{2 i}, \quad k_{j}=\frac{\alpha_{j}}{2 \pi i}, \quad j=1,2, \\
& \tau_{12}=\frac{A_{12}}{2 \pi},
\end{aligned}
$$

where $\sigma_{j}, \alpha_{j}, j=1,2$, and $A_{12}$ are given in (2.4). Then the two-periodic solution (4.5) tends to the two-soliton solution (2.4), that is,

$$
u \rightarrow u_{2}, \quad v \rightarrow v_{2}, \quad \text { as } \rho_{11}, \rho_{22} \rightarrow 0 .
$$

Proof We expand the periodic function $f$ in the following form:

$$
\begin{aligned}
f= & +i\left(e^{2 \pi i \xi_{1}}-e^{-2 \pi i \xi_{1}}\right) e^{-\pi \tau_{11}}+i\left(e^{2 \pi i \xi_{2}}-e^{-2 \pi i \xi_{2}}\right) e^{-\pi \tau_{22}}, \\
& -\left(e^{2 \pi i\left(\xi_{1}+\xi_{2}\right)}+e^{-2 \pi i\left(\xi_{1}+\xi_{2}\right)}\right) e^{-\pi\left(\tau_{11}+2 \tau_{12}+\tau_{22}\right)}+\cdots
\end{aligned}
$$

It follows from (4.6) that

$$
\begin{aligned}
f= & 1+i e^{\xi_{1}^{\prime}}+i e^{\xi_{2}^{\prime}}-e^{\xi_{1}^{\prime}+\xi_{2}^{\prime}-2 \pi \tau_{12}}-i \rho_{11}^{4} e^{-\xi_{1}^{\prime}}-i \rho_{22}^{4} e^{-\xi_{2}^{\prime}} \\
& -\rho_{11}^{4} \rho_{22}^{4} e^{-\xi_{1}^{\prime}-\xi_{2}^{\prime}-2 \pi \tau_{12}}+\cdots \\
\rightarrow & 1+i e^{\xi_{1}^{\prime}}+i e^{\xi_{2}^{\prime}}-e^{\xi_{1}^{\prime}+\xi_{2}^{\prime}+A_{12}}, \\
f^{*} & \rightarrow 1-i e^{\xi_{1}^{\prime}}-i e^{\xi_{2}^{\prime}}-e^{\xi_{1}^{\prime}+\xi_{2}^{\prime}+A_{12}}, \quad \text { as } \rho_{11}, \rho_{22} \rightarrow 0,
\end{aligned}
$$

in which $\xi_{j}^{\prime}=2 \pi i \xi_{j}-\pi \tau_{j j}=\alpha_{j} x+2 \pi i l_{j} t+\sigma_{j}, j=1,2$. Thus the two-periodic solution equation (4.5) tends to the two-soliton solution equation (2.4) provided that

$$
c \rightarrow 0, \quad 2 \pi i l_{j} \rightarrow-\alpha_{j}^{3}, \quad j=1,2, \quad \text { as } \rho_{11}, \rho_{22} \rightarrow 0 .
$$

Assume the solution of system (4.4) is in the following form:

$$
\left(\begin{array}{l}
l_{1} \\
l_{2} \\
u_{0} \\
c
\end{array}\right)=\left(\begin{array}{l}
l_{1}^{(0)} \\
l_{2}^{(0)} \\
u_{0}^{(0)} \\
c^{(0)}
\end{array}\right)+\left(\begin{array}{l}
l_{1}^{(1)} \\
l_{2}^{(1)} \\
u_{0}^{(1)} \\
c^{(1)}
\end{array}\right) \rho_{11}+\left(\begin{array}{l}
l_{1}^{(2)} \\
l_{2}^{(2)} \\
u_{0}^{(2)} \\
c^{(2)}
\end{array}\right) \rho_{22}+o\left(\rho_{11}, \rho_{22}\right)
$$

Expanding the coefficient of system (4.4) and substituting (4.7) into (4.4), together with taking $u_{0}^{(0)}=0$, we then obtain

$$
c^{(0)}=0, \quad 2 \pi i l_{j}^{(0)}+(2 \pi i)^{3} k_{j}^{3}=0, \quad j=1,2, \quad \text { as } \rho_{11}, \rho_{22} \rightarrow 0 .
$$


Thus we have

$$
u_{0} \rightarrow 0, \quad c \rightarrow 0, \quad 2 \pi i l_{j} \rightarrow 8 \pi^{3} k_{j}^{3} i=-\alpha_{j}^{3}, \quad j=1,2, \quad \text { as } \rho_{11}, \rho_{22} \rightarrow 0 .
$$

Therefore we conclude that the two-periodic solution (4.5) tends to the two-soliton solution (2.4) as $\rho_{11}, \rho_{22} \rightarrow 0$.

\section{Conclusions}

In this paper, the Kersten-Krasil'shchik coupled KdV-mKdV system is investigated. Based on the Hirato bilinear method and the Riemann theta function, the one-periodic and twoperiodic wave solutions for the system are constructed. In addition, the asymptotic properties of the quasi-periodic wave solutions are proved. It is shown that the quasi-periodic wave solutions converge to the soliton solutions in a small amplitude limit. It is natural to ask whether we can get multiperiodic wave solutions in the case of $N>2$. However, the solvability of system (2.10) is the key to the construction of multiperiodic wave solutions. As the number of unknown parameters is less than the number of equations, we cannot get multiperiodic wave solutions directly when $N>2$. How to get multiperiodic wave solutions when $N>2$ ? Such a question will be investigated in the future.

Competing interests

The authors declare that no conflict of interest exists in the submission of this manuscript.

Authors' contributions

The manuscript was written through contributions of all authors. All authors have given approval to the final version of the manuscript.

\section{Author details}

${ }^{1}$ College of Science, China University of Mining and Technology, Xuzhou, 221116, P.R. China. ${ }^{2}$ School of Resources and Geosciences, China University of Mining and Technology, Xuzhou, 221116, P.R. China.

\section{Acknowledgements}

This work was supported by the Fundamental Research Funds for the Central Universities (no. 2014QNB50).

Received: 7 May 2016 Accepted: 27 June 2016 Published online: 11 July 2016

\section{References}

1. Ablowitz, MJ, Clarkson, PA: Soliton Nonlinear Evolution Equations and Inverse Scatting. Cambridge University Press, New York (1991)

2. Matveev, VB, Salle, MA: Darboux Transformation and Solitons. Springer, Berlin (1991)

3. Gu, CH, Hu, HS, Zhou, ZX: Darboux Transformation in Soliton Theory and Its Geometric Applications. Science and Technology Press, Shanghai (1999)

4. Hirota, R: The Direct Method in Soliton Theory. Cambridge University Press, New York (2004)

5. Belokolos, ED, Bobenko, Al, Enolskij, VZ, Its, AR, Matveev, VB: Algebro-Geometric Approach to Nonlinear Integrable Equations. Springer, Berlin (1994)

6. Ma, WX, Huang, TW, Zhang, Y: A multiple exp-function method for nonlinear differential equations and its application. Phys. Scr. 82, 065003 (2010)

7. Ma, WX, Zhu, ZN: Solving the (3+1)-dimensional generalized KP and BKP equations by the multiple exp-function algorithm. Appl. Math. Comput. 218, 11871-11879 (2012)

8. Ma, WX: Generalized bilinear differential equations. Stud. Nonlinear Sci. 2, 140-144 (2011)

9. Ma, WX: Bilinear equations and resonant solutions characterized by Bell polynomials. Rep. Math. Phys. 72, 41-56 (2013)

10. Ma, WX, Fan, EG: Linear superposition principle applying to Hirota bilinear equations. Comput. Math. Appl. 61, 950-959 (2011)

11. Nakamura, A: A direct method of calculating periodic wave solutions to nonlinear evolution equations. I. Exact two-periodic wave solution. J. Phys. Soc. Jpn. 47, 1701-1705 (1979)

12. Nakamura, A: A direct method of calculating periodic wave solutions to nonlinear evolution equations. II. Exact oneand two-periodic wave solution of the coupled bilinear equations. J. Phys. Soc. Jpn. 48, 1365-1370 (1980)

13. Fan, EG, Hon, YC: Quasi-periodic waves and asymptotic behavior for Bogoyavlenskiis breaking soliton equation in $(2+1)$ dimensions. Phys. Rev. E 78, 036607 (2008)

14. Hon, YC, Fan, EG, Qin, ZY: A kind of explicit quasi-periodic solution and its limit for the TODA lattice equation. Mod. Phys. Lett. B 22, 547-553 (2008) 
15. Fan, EG: Quasi-periodic waves and an asymptotic property for the asymmetrical Nizhnik-Novikov-Veselov equation. J. Phys. A, Math. Theor. 42, 095206 (2009)

16. Fan, EG, Chow, KW: On the periodic solutions for both nonlinear differential and difference equations: a unified approach. Phys. Lett. A 374, 3629-3634 (2010)

17. Ma, WX, Zhou, RG: Exact one-periodic and two-periodic wave solutions to Hirota bilinear equations in (2 + 1)-dimensional. Mod. Phys. Lett. A 24, 1677-1688 (2009)

18. Zhang, Y, Cheng, ZL: Riemann theta function periodic wave solutions for the variable-coefficient mKdV equation. Chin. Phys. B 21, 120203 (2012)

19. Kersten, P, Krasil'shchik, J: Complete integrability of the coupled KdV-mKdV system. Adv. Stud. Pure Math. 89, 151-171 (2000)

20. Kalkanli, AK, Sakovich, SY, Yurdusen, I: Integrability of Kersten-Krasil'shchik coupled KdV-mKdV equations: singularity analysis and Lax pair. J. Math. Phys. 44, 1703-1708 (2003)

21. Hon, YC, Fan, EG: Solitary wave and doubly periodic wave solutions for the Kersten-Krasil'shchik coupled KdV-mKdV system. Chaos Solitons Fractals 19, 1141-1146 (2004)

22. Qin, Y, Gao, YT, Yu, X, Meng, GQ: Bell polynomial approach and N-soliton solutions for a coupled KdV-mKdV system. Commun. Theor. Phys. 58, 73-78 (2012)

23. Rauch, HE, Farkas, HM: Theta Functions with Applications to Riemann Surfaces. William \& Wilkins, Baltimore (1974)

24. Farkas, HM, Kra, I: Riemann Surfaces. Springer, New York (1992)

\section{Submit your manuscript to a SpringerOpen ${ }^{\mathcal{O}}$ journal and benefit from:}

- Convenient online submission

- Rigorous peer review

- Immediate publication on acceptance

- Open access: articles freely available online

- High visibility within the field

Retaining the copyright to your article 